\title{
COMPOSICIÓN Y VARIACIÓN TEMPORAL DE LA COMUNIDAD DE INSECTOS ACUÁTICOS (INSECTA) EN LA QUEBRADA SARDINEROS, AFLUENTE RIO VERDE, ALTO CAUCA, COLOMBIA
}

\author{
COMPOSITION AND TEMPORAL VARIATION OF AQUATIC INSECT COMMUNITY \\ (INSECTA) IN SARDINEROS CREEK, VERDE RIVER DRAINAGE, UPPER CAUCA, COLOMBIA
}

Carlos A. García-Alzate, César Román-Valencia, Melissa I. González, Ana M. Barrero.

Programa de Bilogía Universidad del Quindío.

Fecha de recibido: Febrero 3 de 2010

Fecha de aceptado: Junio 9 de 2010

Correspondencia: Universidad del Quindío, Laboratorio de Ictiología, A. A. 2639, Armenia, Quindío, Colombia.

Correo electrónico: cagarcia@uniquindio.edu.co.

\section{RESUMEN}

Se analiza la composición y la variación temporal de las comunidades de insectos acuáticos presentes en la parte baja de la quebrada Sardineros, afluente de río Verde, sistema rio Quindío en el Alto Cauca, Colombia. Se colectaron 2743 individuos en bajas lluvias y 1020 en altas lluvias. Diptera y Trichoptera fueron los más abundantes en ambos periodos. Los índices de diversidad de Shannon-Wiener, dominancia de Simpson fueron bajas en ambos periodos climáticos, mientras la riqueza de Margaleffue alta. Los índices indicaron que la diversidad fue baja como consecuencia de la disminución del oxígeno disuelto y el aumento de la conductividad, durante el periodo de altas Iluvias, lo cual contribuyo a la disminución de la calidad del agua que paso de ser buena (BMWP/Col: 191) durante las bajas Iluvias a aceptable (BMWP/Col: 65) en altas lluvias. La prueba Hotelling's T-Squared corroboró las diferencias significativas en la estructura de la comunidad entre épocas $(P=0,042, F=4,6)$ y la prueba de proporciones explico el cambio en la estructura del $74 \%$ de las familias de insectos analizadas; a partir de estos resultados se propone que la estacionalidad fue la variable que mas influyo en abundancia y diversidad de insectos acuáticos.

Palabras clave: Estacionalidad, ecosistema acuático, macroinvertebrados, indicadores biológicos.

\begin{abstract}
We reviewed the composition and variation of aquatic insects from Sardineros Creek, a tributary of Verde river, Quindío river, Upper Cauca, Colombia. 2743 specimens were collected in low rainfall and 1020 during high rainfall. Diptera and Trichoptera were the most abundant. Diversity indices of Shannon-Wiener, Simpson's dominance were low in both climatic periods, while the Margalef richness was high. The index indicated a low diversity as a result of the reduced dissolved oxygen and increased conductivity during the period of high rainfall, which made the declining water quality as good (BMWP/Col: 191) during the low rainfall to acceptable (BMWP/Col: 65) at the high rainfall. Hotelling's T-Squared test probe significative differences between seasons in the community structure $(P=0,042, F=4,6)$ and proportion test explain the structure change in $74 \%$ of the families analyzed; we propose that seasonality was the variable that most influences in the abundance and diversity of aquatic insects.
\end{abstract}

Key words: Seasonality, aquatic ecosystem, macroinvertebrates, biological indicators.

\section{INTRODUCCIÓN}

El ecosistema acuático es el resultado de la interacción de los organismos que allí habitan con la calidad fisicoquímica del agua, la atmósfera y el medio terrestre que los rodea (1). En los últimos años, estos ecosistemas han sido sometidos a numerosas perturbaciones causadas por las actividades humanas; siendo la deforestación, la minera, descarga de residuos sólidos y líquidos de origen domestico e industrial las fuentes de perturbación o contaminación, y la principal causa de cambios en la 
estructura y funcionamiento de las comunidades biológicas $(15,16)$.

Los macroinvertebrados acuáticos han sido uno de los grupos más usados en los estudios de bioindicación, por su capacidad natural de responder a los efectos de perturbaciones eventuales o permanentes, es decir, estas comunidades cambian su estructura y funcionamiento al modificar las condiciones ambientales de sus hábitats (2). La preferencia por éste grupo se debe: 1- son relativamente sedentarios y representativos del área donde son colectados, 2tienen ciclos de vida relativamente cortos comparado con otros organismos y reflejan con mayor rapidez las alteraciones del medio ambiente, 3- viven y se alimentan en o sobre los sedimentos donde tienden acumularse las toxinas, las cuales se incorporan a la cadena trófica a través de estos, 4- su sensibilidad a los factores de perturbación y 5 - son fuente primaria de alimento para muchos peces u otros vertebrados $y$ participan de manera importante en la degradación de la materia orgánica y el ciclo de nutrientes (3), éstas características brindan amplias ventajas en la bioindicación de los cuerpos de agua, dado que permite integrar los efectos de los impactos de diferentes factores de perturbación, acumular en el tiempo el efecto de las tensiones que le han afectado y ser sensibles al impacto de diferentes factores no puntuales que no pueden ser detectados por otros métodos (4).

En este sentido, el objetivo de este estudio fue evaluar la composición y variación temporal de la comunidad de insectos acuáticos, además, determinar la calidad del agua en la parte baja de la quebrada Sardineros, afluente del Rio Verde, Rio Quindío, Alto Cauca, Colombia.

\section{MATERIAL Y MÉTODOS}

\section{Área de estudio}

El trabajo se realizó en la parte baja de la quebrada Sardineros, afluente del río Verde, Rio Quindío, ubicada al suroccidente del departamento del Quindío a 4o $23^{\prime} 00^{\prime \prime \prime} \mathrm{N}$ y 75ㅇ 45'00"O, a $1249 \mathrm{msnm}$. La quebrada Sardineros se caracteriza por presentar un sustrato de tipo rocoso-arenoso, la vegetación riparía de Guadua angustifolia y pastos (Poaceae) predominantemente, además, de Guayabos (Myrtaceae), Plátanos (Musaceae) y Heliconeas (Heliconaceae).
Los muestreos se llevaron a cabo del 29 al 30 de agosto y del 17 al 18 de octubre del 2008, teniendo en cuenta los registros de pluviosidad del IDEAM para abarcar los periodos de bajas y altas lluvias de la zona (Figura 1).

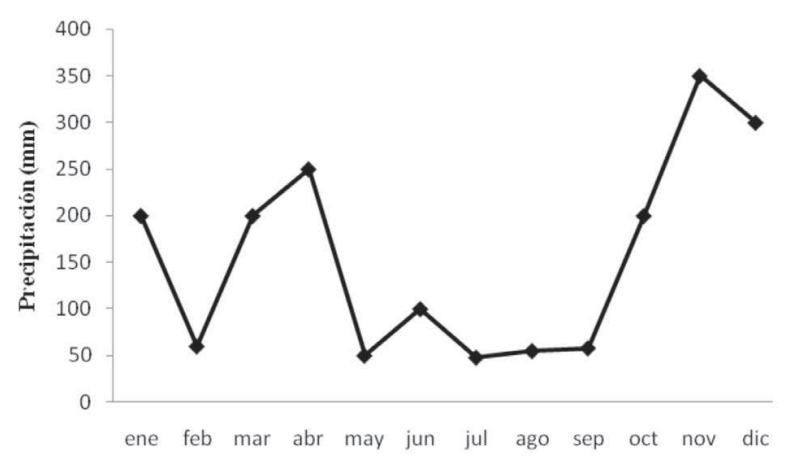

Figura 1. Registros de precipitación, esstación Río Lejos, Pijao, Quindío. Datos del IDEAM.

\section{Variables fisicoquímicas}

In situ se registraron oxigeno disuelto, porcentaje de saturación y temperatura superficial del agua con un oxímetro OXI196-microprocesador; conductividad con conductímetro HANNA HI98842, pH con potenciómetro PIN POINT-BNC y temperatura del aire con termómetro de mercurio. Para determinar las variables químicas como durezas totales, sólidos (disueltos, suspendidos y totales), demanda bioquímica de oxigeno (D.B.O) y demanda química de oxigeno (D.Q.O) se tomaron muestras de agua en frascos plásticos de un litro de capacidad de acuerdo con la metodología recomendada por (5) y (6); estas determinaciones se realizaron en el laboratorio de aguas de la Universidad del Quindío en Armenia.

\section{Variables biológicas}

Los muestreos se llevaron a cabo en 300 metros corriente arriba y abajo de flujo de la quebrada Sardineros. La recolecta se realizó de forma indirecta por medio de una red Surber y una red de pantalla y de forma directa con pinzas entomológicas al obtener los organismos presentes en rocas, troncos y hojarasca; los ejemplares fueron fijados en frascos con alcohol al $70 \%$ debidamente rotulados.

Las muestras se trasladaron al Laboratorio de Biología de la Universidad del Quindío, para su determinación hasta el taxón más bajo posible, por medio de claves, listas, ilustraciones $(7 ; 8 ; 6 ; 9)$.

Rev. Invest. Univ. Quindío (21): 21- 28. Armenia - Colombia 


\section{Análisis estadístico}

Se calculo la abundancia relativa (A.R \%.) para cada grupo de insectos acuáticos en cada periodo climático; se realizó una prueba de Hotelling's T-Squared en el programa SPSS Statistic 17,0 bajo Windows con $95,0 \%$ de confiabilidad, para evaluar las diferencias en la estructura de las comunidades en los dos periodos climáticos, además se realizó una prueba de proporciones en el programa STAGRAPHIC 5,1 con un p $>0,05$ y $95,0 \%$ de confiabilidad, para dilucidar cuales familias presentaban cambios en su estructura. Para describir la estructura numérica de la comunidad de macro invertebrados, se utilizaron los siguientes de diversidad de Shannon-Wiener (10), dominancia de Simpson (11) y riqueza de Margalef (12).

Para determinar el estado ecológico de la quebrada se evalúo el Biological Monitoring Working Party modificado para Colombia (BMWP/Col) (13), puntuación promedio por taxón (Average Score Per Taxón, ASPT), relación y porcentaje de Ephemeroptera, Plecoptera y Trichoptera (EPT y \%EPT, respectivamente).

\section{RESULTADOS}

\section{Variables fisicoquímicas}

El área de estudio se caracterizó por presentar una temperatura ambiente de 21,17 ㅇ C en bajas lluvias y de 20,31 $9 \mathrm{C}$ en altas Iluvias; la temperatura del agua presento valores de 20,09 y $19,3 \circ \mathrm{O}$ en bajas y altas Iluvias respectivamente. En bajas Iluvias el oxigeno disuelto $(7,03 \mathrm{mg} / \mathrm{l})$ y el porcentaje de saturación de oxigeno (80\%) fueron más altos que para altas Iluvias (3,69 $\mathrm{mg} / \mathrm{l}$ y $45 \%$ respectivamente); mientras el $\mathrm{pH}$ $(7,95)$, la conductividad $(2,79 \mu \mathrm{s} / \mathrm{cm})$, las durezas totales $(29,6 \mathrm{mg} / \mathrm{CaCO} 3)$, los sólidos totales (188 $\mathrm{mg} / \mathrm{l}), \operatorname{DBO}(4,8 \mathrm{mg} / \mathrm{l} \mathrm{O2})$ y $\mathrm{DQO}(4,8 \mathrm{mg} / \mathrm{l} \mathrm{O} 2)$ presentaron un aumento en Iluvias $(8,21 ; 3,82 \mu \mathrm{s} / \mathrm{cm}$; $44 \mathrm{mg} / \mathrm{l} \mathrm{CaCO} 3 ; 211,14 \mathrm{mg} / \mathrm{l} ; 17,7 \mathrm{mg} / \mathrm{l} 02$ y $25,2 \mathrm{mg} / \mathrm{l}$ O2 respectivamente) (Tabla 1 ).

Tabla 1. Variables fisicoquímicas para la parte baja de la quebrada Sardineros, afluente río Verde, Alto Cauca, Colombia.

\begin{tabular}{|c|c|c|}
\hline Variable & Bajas L luvias & Altas L luvias \\
\hline Temperatura ambiente $(\stackrel{\circ}{ } \mathrm{C})$ & 21,17 & 20,31 \\
\hline Temperatura del agua (으) & 20,09 & 19,3 \\
\hline Oxigeno disuelto (mg/) & 7,03 & 3,69 \\
\hline Porcentaje de saturación oxigeno( \% ) & 80 & 45 \\
\hline $\mathrm{pH}$ & 7,95 & 8,21 \\
\hline Conductividad ( $\mu \mathrm{s} / \mathrm{cm})$ & 2,79 & 3,82 \\
\hline D ureza total (mg/CaC O3) & 29,6 & 44 \\
\hline Sólidos totales (mg/) & 188 & 211,14 \\
\hline Sólidos disueltos (mg/) & 185 & 208 \\
\hline Sólidos suspendidos (mg/) & 3 & 3,14 \\
\hline D.Q.O $\left(\mathrm{mg} / \mathrm{O}_{2}\right)$ & 4,8 & 25,2 \\
\hline D.B.O (mg/ $\left.\mathrm{O}_{2}\right)$ & 4,8 & 17,7 \\
\hline
\end{tabular}

\section{Variables biológicas}

Se recolectó un total de 3763 individuos; 2743 (73\%) en bajas lluvias y 1020 (27\%) en altas lluvias; representados en 8 ordenes 27 familias y 37 géneros (Figura 2, Tabla 2). En bajas Iluvias Diptera fue el grupo más representativo con 1204 ejemplares $(43,89 \%$ A.R), 4 familias y 5 géneros; Blepharoceridae fue la familia más abundante con 884 ejemplares pertenecientes a 2 géneros; Paltostoma y Limonicola, siendo Paltostoma el género más representativo con 478 individuos; sigue Trichoptera con 545 individuos 
$(19,87 \%$ A.R) representado en 4 familias y 5 géneros; Leptonema, Smicridea, Atopsyche, Helicopsyche Mortoniella. Hydropsychidae fue la familia más numerosa con 406 ejemplares y el género Leptonema con 290 individuos el más representativo. En lluvias el grupo menos abundante fue Neuroptera con 57 individuos $(2,08 \%)$ de la familia Corydalidae, genero Corydalus, seguido de Odonata con 61 individuos $(2,22 \%$ A.R). En altas lluvias el orden más representativo fue Trichoptera con 382 individuos (37,45\% A.R), Hydropsychidae y Leptonema fueron los más abundantes con 326 y 198 individuos respectivamente; en este periodo las familias Hydrobiosidae y Glossomatidae no fueron colectadas. Diptera representado por 302 individuos (29,61\% A.R) pertenecientes a 2 familias; Simuliidae, género Simulium con 192 individuos y Blepharoceridae, genero Limonicola con 110 ejemplares. El orden menos abundante fue Neuroptera con 22 individuos (2,16\% A.R), familia Corydalidae, género Corydalus.

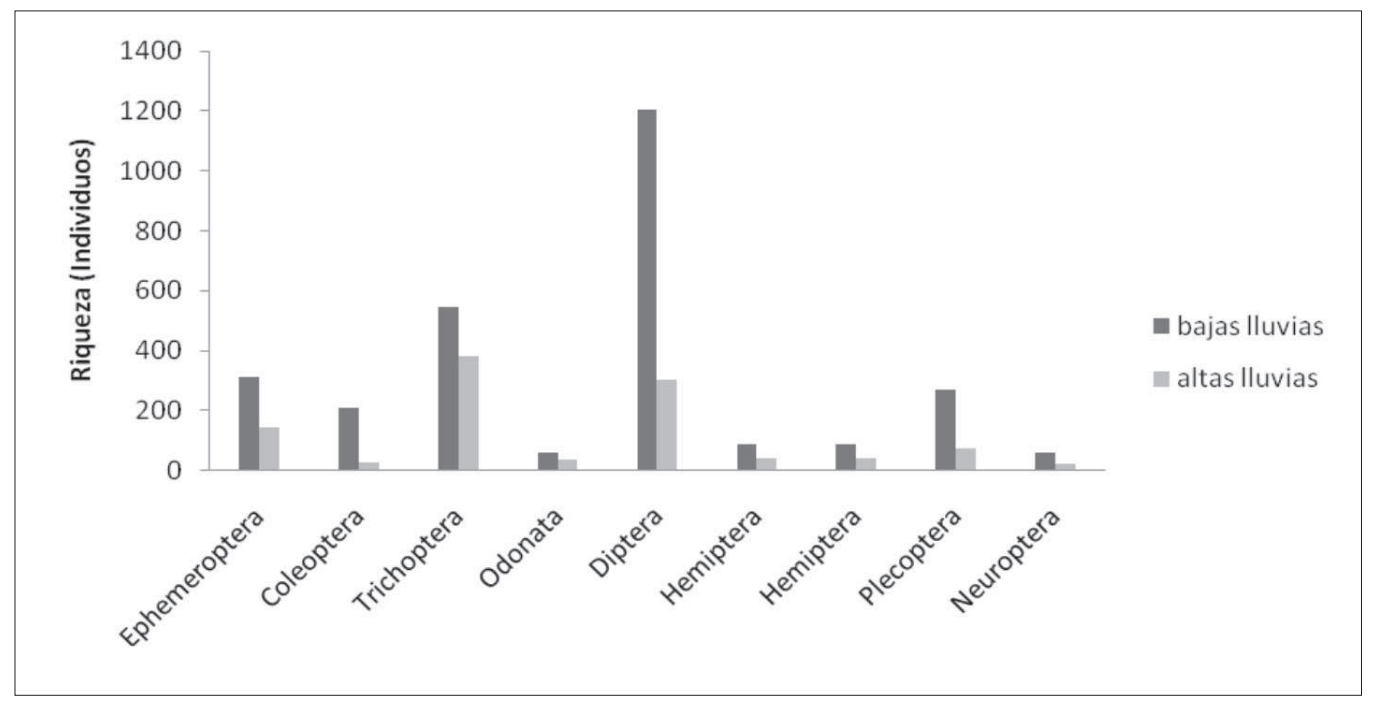

Figura 2. Abundancia en órdenes de insectos acuáticos durente altas y bajas lluvias en la parte baja de la quebrada Sardineros, afluente río Verde, Alto Cauca, Colombia 
García-Alzate, C. A., Román-Valencia, C., González, M. I., Barrero, A. M. - 25-

Tabla 2. Abundancia de insectos acuáticos en la parte baja de la quebrada Sardineros, afluente río Verde, Alto Cauca, Colombia. A.B = Abundancia absoluta.; A.R = Abundancia relativa.

\begin{tabular}{|c|c|c|c|c|c|c|}
\hline \multirow[b]{2}{*}{ Orden } & \multirow[b]{2}{*}{ F amilia } & \multirow[b]{2}{*}{ G énero } & \multicolumn{2}{|c|}{ A.B } & \multicolumn{2}{|c|}{ A.R.\% } \\
\hline & & & $\begin{array}{c}\text { Bajas } \\
\text { L luvias }\end{array}$ & $\begin{array}{c}\text { Altas } \\
\text { L luvias }\end{array}$ & $\begin{array}{c}\text { Bajas } \\
\text { L luvias }\end{array}$ & $\begin{array}{c}\text { Altas } \\
\text { L luvias } \\
\end{array}$ \\
\hline \multirow[t]{8}{*}{ E phemeroptera } & B aetidae & Baetodes & 135 & 18 & 4,92 & 1,76 \\
\hline & & Nanomis & 1 & 2 & 0,04 & 0,2 \\
\hline & & Camelobaetidius & 32 & 2 & 1,17 & 0,2 \\
\hline & & Mayobaetis & 1 & & 0,04 & \\
\hline & L eptohyphidae & Leptohyphes & 33 & 62 & 1,2 & 6,08 \\
\hline & L eptophlebiidae & Tricorythodes & 2 & 8 & 0,07 & 0,78 \\
\hline & & Thraulodes & 104 & 44 & 3,79 & 4,31 \\
\hline & Oligoneuriidae & Lachlania & 5 & 5 & 0,18 & 0,49 \\
\hline \multirow[t]{5}{*}{ Coleoptera } & Hydrophilidae & Berosus & 73 & & 2,66 & \\
\hline & Staphylinidae & Stenus & 2 & & 0,07 & \\
\hline & Psephenidae & Psephenops & 8 & 8 & 0,29 & 0,78 \\
\hline & Elmidae & Heterelmis & 123 & 7 & 4,48 & 0,69 \\
\hline & Ptilodactylidae & Anchytarsus & 3 & 11 & 0,11 & 1,08 \\
\hline \multirow[t]{5}{*}{ Trichoptera } & Hydropsychidae & Leptonema & 290 & 198 & 10,57 & 19,41 \\
\hline & & Smicridea & 116 & 128 & 4,23 & 12,55 \\
\hline & Hydrobiosidae & Atopsyche & 15 & & 0,55 & \\
\hline & Helicopsychidae & Helicopsyche & 114 & 56 & 4,16 & 5,49 \\
\hline & Glossosomatidae & Mortoniella & 10 & & 0,36 & \\
\hline \multirow[t]{5}{*}{ Odonata } & Libellulidae & Brechmorhoga & 43 & 26 & 1,57 & 2,55 \\
\hline & Gomphidae & Progomphus & 4 & 1 & 0,15 & 0,1 \\
\hline & & Phyllogomphosdes & 6 & 1 & 0,22 & 0,1 \\
\hline & Calopterygidae & Hetaerina & 3 & 1 & 0,11 & 0,1 \\
\hline & A eshnidae & Aeshna & 5 & 5 & 0,18 & 0,49 \\
\hline \multirow[t]{5}{*}{ Diptera } & Simuliidae & Simulium & 285 & 192 & 10,39 & 18,82 \\
\hline & Psychodidae & Clognia & 1 & & 0,04 & \\
\hline & Blepharoceridae & Limonicola & 406 & 110 & 14,8 & 10,78 \\
\hline & & Paltostoma & 478 & & 17,43 & \\
\hline & Tipulidae & Tipula & 34 & & 1,24 & \\
\hline \multirow[t]{6}{*}{ Hemiptera } & Naucoridae & Pelocoris & 17 & 27 & 0,62 & 2,65 \\
\hline & & Heleocoris & 5 & & 0,18 & \\
\hline & Veliidae & Rhagovelia & 8 & 2 & 0,29 & 0,2 \\
\hline & Guerridae & Brachymetra & 51 & 9 & 1,86 & 0,88 \\
\hline & & Trepobates & 3 & & 0,11 & \\
\hline & B elostomatidae & Belostoma & 1 & & 0,04 & \\
\hline \multirow[t]{2}{*}{ Plecoptera } & Perlidae & Anacroneura & 263 & 75 & 9,59 & 7,35 \\
\hline & & Perlodes & 6 & & 0,22 & \\
\hline Neuroptera & Corydalidae & Corydalus & 57 & 22 & 2,08 & 2,16 \\
\hline TOTAL & & & 2743 & 1020 & 100 & 100 \\
\hline
\end{tabular}

Rev. Invest. Univ. Quindío (21): 21- 28. Armenia - Colombia 


\section{- 26 - Composición y variación temporal de la comunidad insectos acuáticos}

La prueba Hotelling's T-Squared corroboró las diferencias significativas en la estructura de la comunidad para las bajas y altas lluvias $(P=0,042$, $\mathrm{F}=4,6)$. La prueba de proporciones arrojó valores menores a 0,05 para el $74 \%$ de las familias analizadas, lo cual explica el cambio en la estructura de 20 familias, mientras que para el $26 \%$ de las familias la prueba arrojó valores mayores a 0,05; por lo tanto en 7 familias (Leptophlebiidae, Oligoneuriidae, Psephenidae, Hydropsychidae, Simuliidae, Naucoridae y Aeshnidae) no se encontraron diferencias significativas (Tabla 3).

Tabla 3. Prueba de proporciones para insectos acuáticos encontrados en la parte baja de la quebrada Sardineros, afluente río Verde,AltoCauca,Colombia.

\begin{tabular}{|c|c|c|}
\hline Orden & Familia & P-Valor \\
\hline \multirow[t]{3}{*}{ E phemeroptera } & B aetidae & $7,60 \mathrm{E}-08$ \\
\hline & L eptophlebiidae & 0,72405 \\
\hline & Oligoneuriidae & 0,72405 \\
\hline \multirow[t]{5}{*}{ Coleoptera } & Hydrophilidae & $1,92 E-10$ \\
\hline & Staphylinidae & $1,92 E-10$ \\
\hline & Psephenidae & 0,72405 \\
\hline & Elmidae & $8,75 E-09$ \\
\hline & Ptilodactylidae & $1,403 E-05$ \\
\hline \multirow[t]{4}{*}{ Trichoptera } & Hydropsychidae & 0,72405 \\
\hline & Hydrobiosidae & $1,92 \mathrm{E}-10$ \\
\hline & Helicopsychidae & 0,0416224 \\
\hline & Glossosomatidae & $1,92 \mathrm{E}-10$ \\
\hline \multirow[t]{4}{*}{ Odonata } & Libellulidae & 0,180647 \\
\hline & Gomphidae & $2,5357 E-05$ \\
\hline & Calopterygidae & 0,00169848 \\
\hline & A eshnidae & 0,72405 \\
\hline \multirow[t]{4}{*}{ Diptera } & Simuliidae & 0,290136 \\
\hline & Psychodidae & $1,92 E-10$ \\
\hline & Blepharoceridae & $5,93 E-07$ \\
\hline & Tipulidae & $1,92 \mathrm{E}-10$ \\
\hline \multirow[t]{4}{*}{ Hemiptera } & Naucoridae & 0,290136 \\
\hline & Veliidae & 0,00013732 \\
\hline & Guerridae & $4,1197 E-06$ \\
\hline & B elostomatidae & $1,92 \mathrm{E}-10$ \\
\hline Plecoptera & Perlidae & 0,00039322 \\
\hline Neuroptera & Corydalidae & 0,00636545 \\
\hline
\end{tabular}

El índice de diversidad Shannon-Weaner fue bajo en ambos periodos climáticos (Tabla 4), con un valor de 2,52 y 2,84 en altas y bajas Iluvias respectivamente. La dominancia de Simpson indicó baja dominancia en ambas épocas, sin embargo, en altas lluvias el valor fue mayor que en bajas Iluvias $(0,18$ y 0,09 respectivamente). La riqueza de Margalef fue alta con 5,55 y 3,75 para bajas y altas lluvias respectivamente, como consecuencia de una mayor dominancia de grupos.

Tabla 4. Índices de diversidad para las comunidades de insectos acuáticos en la parte baja de la quebrada Sardineros, afluente río Verde,AltoCauca,Colombia.

\begin{tabular}{lcc}
\hline \multicolumn{1}{c}{ Índice } & B ajas L luvias & Altas L luvias \\
\hline Shannon-Weaner & 2,84 & 2,52 \\
Simpson & 0,08 & 0,18 \\
Margalef & 5,54 & 3,75 \\
\hline
\end{tabular}

La comunidad de Ephemeropteros, Plecopteros y Trichopteros (EPT) se encontró representada por un total de 1142 individuos (\%EPT: 41,05) en bajas Iluvias y 598 (\%EPT: 58,62) en altas lluvias; la abundancia de estos organismos bajó como respuesta a los cambios en la calidad del agua, la pasó de ser de buena (BMWP/Col: 191) a aceptable (BMWP/Col: 65); este valor se asocia con el ASPT (7,07 en bajas Iluvias y 2,41 en altas Iluvia).

\section{DISCUSIÓN}

La comunidad de insectos acuáticos se encontró dentro de lo registrado para otros drenajes del alto Cauca $(14 ; 15 ; 16)$, se observó alta riqueza de organismos que varían de acuerdo a la escala temporal, siendo ésta la variable que más influyó en el número de individuos recolectados. La disminución de la abundancia en el periodo de altas lluvias se relaciona con las perturbaciones ambientales provocadas por la reducción de agregación de organismos debido al nivel que alcanza el agua en esta época, ya que tanto la biomasa como la diversidad de macrobentos están fuertemente influenciadas por la variación en el nivel de las aguas $(1 ; 17)$.

En la Quebrada Sardineros la entrada de energía, la heterogeneidad, los disturbios ambientales y las interacciones bióticas fueron importantes elementos que determinaron la diversidad de las comunidades de insectos acuáticos; entre los factores bióticos se incluyó la cobertura de la vegetación riparia, la cual contribuyó a aumentar la cantidad de material alóctono que llega al cuerpo de agua y favorece la aparición de refugios para los insectos y por ende el incremento de la fauna $(18 ; 19)$. Así mismo las 
interacciones abióticas son un factor importante que puede favorecer o limitar la abundancia de algunas especies, entre estos factores se incluyen las variables fisicoquímicas a partir de las cuales las comunidades cambian su estructura. El oxigeno disuelto es una de las variables fisicoquímicas que limitan la distribución y estructura de los insectos acuáticos, como se observó en la comunidad de la quebrada Sardineros ya que se limitó el número de individuos y especies en el momento en que se observaron bajos rangos en esta variable.

El cambio en la estructura de la comunidad de macroinvertebrados de la quebrada Sardineros, permite demostrar que el cuerpo de agua está perturbado por algún tipo de factor contaminante, debido a que son pocas las especies tolerantes a condiciones de baja oxigenación y las pertenecientes a los ordenes Ephemeroptera y Plecoptera son los primeros en desaparecer debido a que estos habitan en ambientes con altos niveles de oxigeno disuelto (13).

El aumento de los sólidos disueltos y la turbidez fueron variables limitantes para el establecimiento de la fauna de macroinvertberados en la quebrada Sardineros, dado que estos impiden el paso de rayos solares, dañan y taponan el sistema de intercambio gaseoso en los animales acuáticos y destruyen sus hábitats naturales (1); afectan directamente a las comunidades vegetales que sirven de alimento y refugio para los insectos; lo que representa de esta manera una variable relacionada con la presencia de estos organismos en el ecosistema acuático (19). Se concluye que la estacionalidad es una variable muy importante al analizar la abundancia y diversidad de comunidades acuáticas, esta modifica a su vez las variables fisicoquímicas y biológicas del ecosistema acuático. A partir del análisis de la comunidad de macroinvertebrados, se plantea que en épocas de altas lluvias el cuerpo de agua recibe mayor cantidad de sedimentos, lo que contribuye a la disminución del oxigeno disuelto en el agua y por ende tiene como consecuencia la disminución de la calidad del agua como la diversidad de especies de insectos acuáticos.

\section{AGRADECIMIENTOS}

Se recibió financiamiento por parte de la Universidad del Quindío-Vicerrectoría de Investigaciones (proyecto 357 y 464) y del Programa Académico de Biología. Al grupo de investigación y asesoría en estadística de la Universidad del Quindío, en especial al profesor Hernando Hurtado. A la Bióloga Paola Andrea Villegas por su colaboración en la fase de laboratorio. A los estudiantes de Biología del curso de Limnología (2008-II) por su ayuda durante el trabajo de campo. Este artículo se benefició de críticas y sugerencias de un evaluador anónimo.

\section{BIBLIOGRAFÍA}

1. Roldán, G \& Ramírez, J. J. 2008. Fundamentos de limnología neotropical. 2a edición, Fen-Universidad de Antioquia, Medellín. 440 p.

2. Bonada, N. Prat, N.; Resh, V.H. \& Statzner, B. 2006. Developments in aquatic insect biomonitoring: a comparative analysis of recent approaches. Annual Review Entomolology, 51: 495-523.

3. Reece, P. Y \& Richardson, S. 2000. Biomonitoring with the reference condition approach for the detection of aquatic ecosystem at risk. Pp. 549-552 in L.M. Dearling (ed): Proceedings of a conference on the biology and management of species and habitats at risk, Kamloops, 15-19.

4. Barbour, M. T. J. Gerritsen, B. D. Snyder \& J. B. Stribling. 1999. Rapid bioassessment protocols for use in streams and wadeable rivers: periphyton, benthic macroinvertebrates and fish, second edition. EPA 841-B-99-002. U.S. environmental protection agency; office of water; Washington, D.C. 350 p.

5. Apha, american public health association american waterworks (awwa), water pollution control federation (wpcf). 1998. Standard Methods for examination of Water and Sewage and Wastewater. 20a ed. New York.

6. Wetzel, R. \& Likens, G. 2000. Limnological analyses, 3 ed. Nueva York: Springer-Verlag. 429 p.

7. Roldán, G. 1996. Guía para el estudio de los macroinverterados acuáticos del departamento de Antioquia. FENColciencias. Bogotá. 217 p.

8. Merritt R. W \& Cummins, K. W. 1996. An introduction to the aquatic insects of north America. Kendall/Hunt publishing co. Dubuque, lowa. $862 \mathrm{p}$. 
9. Posada-García J. A, \& Roldán, G. 2003. Clave llustrada y diversidad de las larvas de Tricoptera en nor-occidente de Colombia. Caldasia, 25(1): 169-192.

10. Shannon, C \& Weaver, W. 1949. The Matematical theory of communication, The University of Illinois Press. Urbana, 19-27.

11. Simpson, E. H, 1945. Mesurment of diversity. Nature, 163 (4148): 688 p.

12. Margalef, R. 1951. Diversidad de especies en las comunidades naturales. Inst. Biol. Appl. 9: 15-27.

13. Roldán, G. 2003. Bioindicación de la calidad del agua en Colombia. Uso del método BMWP/Col. FEN Universidad de Antioquia, Medellín, $170 \mathrm{p}$.

14. Londoño, A.; Arrubla J.; Toro J.; Torres D.; Zarate M. \& Beltrán M. 2005. Determinación de la calidad ambiental en la quebrada Agua Bonita, corregimiento de Barcelona, Departamento del Quindío. Revista de InvestigacionesUniversidad del Quindío, 15:55-64.

15. García-Alzate C., Román-Valencia, C., Vanegas-Ríos, A. \& Arcila-Mesa, D. 2007. Análisis fisicoquímico y biológico comparado en dos quebradas de alta montaña neotropical. Revista de Investigaciones-Universidad del Quindío, 17: $57-80$.

16. García-Alzate C., Román-Valencia, C., Lopera, D .X., González, M. I \& Simunovic, M. 2008. Physico-chemical and biological variables of San Jose creek, Otun river drainage/ upper Cauca, Colombia. Revista de InvestigacionesUniversidad del Quindío, 20:38-48.

17. Crisci-Bispo, V. Bispo, P. \& Froehlich, C. 2007. Ephemeroptera, Plecoptera and Trichoptera assemblages in two Atlantic rainforest streams, southeastern Brazil. Revista Brasileira de Zoologia, 24 (2):312-318.

18. Bispo, P \& Oliveira, L. 2007. Diversity and structure of Ephemeroptera, Plecopetra and Trichoptera (Insecta) assemblages from riffles in mountain streams of Central Brazil. Revista Brasileira de Zoologia, 24(2): 283-293.

19. Lomond, T. 1997. Can a naturally impoverished boreal Ephemeroptera, Plecoptera and Trichoptara (EPT) fauna serve as an indicator of water quality?. Tesis doctoral. Department of Biology, Mernorial University of Newfoundland., Canada. 200 p. 